\title{
Competências emocionais: estudo de validação de um instrumento de medida
}

\author{
José Maurício Haas Bueno¹, Fernanda Maria de Lira Correia \\ Mussa Abacar, Yves de Albuquerque Gomes, Francisco Santos Pereira Júnior \\ Universidade Federal de Pernambuco, Recife-PE, Brasil
}

\section{RESUMO}

Este trabalho teve como objetivos a construção e avaliação das propriedades psicométricas de um Inventário de Competências Emocionais (ICE). Os 76 itens do instrumento foram construídos para representar quatro habilidades relacionadas à inteligência emocional: percepção de emoções, uso da emoção para facilitação do pensamento, compreensão de emoções e regulação de emoções. O instrumento foi aplicado a 409 brasileiros adultos, predominantemente do sexo feminino (76,5\%), com idade média de 30,2 anos $(D P=11,5)$, que o responderam em uma escala Likert de cinco pontos. Uma análise fatorial exploratória revelou uma estrutura com cinco fatores: percepção de emoções, regulação de emoções de baixa potência, expressividade emocional, regulação de emoções em outras pessoas e regulação de emoções de alta potência. Além disso, observou-se um padrão de correlações positivas e significativas com diversos aspectos da qualidade de vida. Concluiu-se que o instrumento apresenta condições psicométricas favoráveis para a recomendação de seu uso em pesquisas.

Palavras-chave: inteligência emocional; emoções; qualidade de vida.

\section{ABSTRACT - Emotional competences: study of the validity of a measurement instrument}

This work aimed at the construction and evaluation of the psychometric properties of an Emotional Competence Inventory (ECI). The 76 items of the instrument were constructed to represent four skills related to emotional intelligence: emotional perception, the usage of emotion to facilitating thought, emotional understanding and emotional regulation. The instrument was administered to 409 Brazilian adults, predominantly female $(76.5 \%)$, with a mean age of $30.2(S D=11.5)$, who answered via a five-point Likert scale. An exploratory factor analysis revealed a structure with five factors: emotional perception, regulation of low potency emotions, emotional expressivity, emotional regulation in other people and regulation of high potency emotion. Furthermore, it was observed a pattern of positive and significant correlations with many aspects of quality of life. It was concluded that the instrument has favorable psychometric conditions to recommend its use in research.

Keywords: emotional intelligence; emotions; quality-of-life.

\section{RESUMEN - Competencias emocionales: estudio de validación de un instrumento de medición}

Este trabajo tuvo como objetivo la construcción y evaluación de las propiedades psicométricas de un Inventario de Competencias Emocionales (ICE). Los 76 ítems del instrumento representaban cuatro habilidades relacionadas con la inteligencia emocional: percepción de emociones, uso de emociones para facilitar el pensamiento, comprensión de emociones y regulación de emociones. Se aplicó el instrumento a 409 adultos brasileños, predominantemente mujeres $(76,5 \%)$ con edad media de 30,2 años $(D E=11,5)$ que respondieron a través de una escala Likert de 5 puntos. Un análisis factorial exploratoria reveló una estructura con cinco factores: percepción de emociones, regulación de emociones de baja potencia, expresividad emocional, regulación de emociones en los otros y regulación de emociones de alta potencia. Además, se observó un patrón de correlaciones positivas y significativas con diversos aspectos de la calidad de vida. Se concluyó que el instrumento tiene condiciones psicométricas favorables para recomendar su uso en la investigación.

Palabras clave: inteligencia emocional; emociones; calidad de vida.

O conceito de Inteligência Emocional (IE) foi formalmente introduzido por Salovey e Mayer (1990) como um novo tipo de inteligência, relacionada ao processamento de informações carregadas de afeto. Posteriormente, foi detalhada como a resultante de um conjunto de quatro habilidades: 1. percepção e expressão, que é a capacidade de identificar emoções em si mesmo, em outras pessoas e em condições físicas e ambientais; 2. facilitação emocional do pensamento, que é a capacidade de incluir as emoções no raciocínio para resolver

${ }^{1}$ Endereço para correspondência: Universidade Federal de Pernambuco - UFPE, Centro de Filosofia e Ciências Humanas - CFCH, Programa de Pós-Graduação em Psicologia Cognitiva, $8^{\circ}$ andar, Av. da Arquitetura, s/n Cidade Universitária, 50740-550, Recife-PE. E-mail: mauricio.ufpe@gmail.com 
problemas; 3. compreensão emocional, que é a capacidade de identificar tipos de situações em que as emoções costumam ser eliciadas, discriminar emoções semelhantes e opostas e o que elas transmitem; e 4. regulação de emoções, que envolve o entendimento das implicações de atos sociais sobre as emoções e controle emocional em si mesmo e no outro (Mayer, Roberts, \& Barsade, 2008; Mayer, \& Salovey, 1997; Salovey, \& Grewal, 2005; Salovey, \& Mayer, 1990).

Desde sua proposição, sua pertinência ou não como um novo tipo de inteligência tem sido objeto de muitas discussões na literatura científica (Mayer, Salovey, \& Caruso, 1999; Roberts, Zeidner, \& Matthews, 2001; Woyciekosk, \& Hutz, 2009). Nesse sentido, alguns autores (Hedlund, \& Sternberg, 2002; Mayer, Salovey, \& Caruso, 2000; Petrides, \& Furnham, 2000) têm apontado duas posições no campo conceitual da IE. A primeira, mais cognitiva, é baseada na noção de que a IE é a capacidade de perceber e compreender informações emocionais, de forma a favorecer a condução de comportamentos mais adequados ao contexto. A segunda posição define a inteligência emocional como traço, procurando identificar aqueles que se associam ao êxito ou sucesso na vida, aos quais, por vezes, são incluídas habilidades não cognitivas, como a assertividade, a empatia, o otimismo e construtos tradicionalmente relacionados ao estudo da personalidade.

Ambas as posições, no entanto, estudam as interações entre emoção e inteligência, o que leva à complexidade no campo conceitual, bem como às dificuldades metodológicas decorrentes (Woyciekosk \& Hutz, 2009). Uma dessas dificuldades é o fato de que uma mesma definição de inteligência emocional, como a de Mayer e Salovey (1997), por exemplo, pode ser operacionalizada tanto sob a perspectiva cognitiva, por meio de instrumentos de desempenho (Mayer, Salovey, \& Caruso, 1997; Mayer, Salovey, \& Caruso, 2002; Mayer, Salovey, Caruso, \& Sitarenios, 2003), quanto sob a perspectiva do traço, por meio de instrumentos de autorrelato (Faria et al., 2006; Petrides, \& Furnham, 2000; Schutte et al., 1998).

A abordagem cognitiva, tradicionalmente, tem usado a Multifactor Emotional Intelligence Scale (MEIS; Mayer et al., 1997) e o Mayer-Salovey-Caruso Emotional Intelligence Test (MSCEIT; Mayer et al., 2002; Mayer et al., 2003). Vários estudos foram realizados para buscar evidências de validade e fidedignidade desses instrumentos para avaliação da IE (Mayer et al., 1997; Mayer et al., 1999; Mayer et al., 2003; Palmer, Gignac, Manocha, \& Stough, 2005; Roberts et al., 2001), incluindo estudos realizados no contexto cultural brasileiro (Cobêro, 2004; Dantas, \& Noronha, 2005; Jesus Jr., 2004; Noronha, Primi, Freitas, \& Dantas, 2007; Primi, Bueno, \& Muniz, 2006). Embora esses estudos tenham apresentado resultados encorajadores para o estabelecimento da inteligência emocional como um tipo de inteligência, algumas críticas lhes têm sido feitas. Por exemplo, o sistema de avaliação por concordância impede o desenvolvimento de itens difíceis, dificultando a discriminação das diferenças individuais de sujeitos com altas habilidades em IE (Bueno, \& Primi, 2003; Noronha et al., 2007). Além disso, os subtestes de regulação de emoções são apenas parcialmente de desempenho, já que contemplam uma resposta correta, mas não exigem que a pessoa realmente regule emoções para a formulação da resposta, aproximando-se bastante do formato dos instrumentos de avaliação por autorrelato (Bueno, 2013).

Os instrumentos de autorrelato são uma alternativa aos instrumentos de desempenho, pois permitem que o respondente indique o nível de competência percebida no processamento de informações emocionais, seja ele alto ou baixo. Assim, embora captem mais a autopercepção da capacidade do que a capacidade em si, permitem a discriminação das diferenças individuais em toda a amplitude do construto, superando, nesse aspecto, os instrumentos de desempenho para avaliação da IE. Por esse motivo, e também por não ter sido encontrado um instrumento de autorrelato para avaliação da IE, com base na teoria de Mayer e Salovey, para o contexto cultural brasileiro, este estudo se dedicou a desenvolvê-lo.

Embora Mayer et al. (2000) defendam a avaliação da IE por instrumentos de desempenho, alguns instrumentos de autorrelato foram desenvolvidos, em outras culturas, para avaliá-la, como o Emotional Intelligence Inventory (Schutte et al., 1998) e o Emotional Skills and Competence Questionnaire (ESCQ; Faria et al., 2006). O Emotional Intelligence Inventory (Chico, 1999; Schutte et al., 1998) foi desenvolvido para adultos americanos $(N=346)$, resultando numa escala unifatorial com 33 itens e um bom índice de consistência interna $(\alpha=0,90)$. Contudo, a replicação desse estudo, realizada por Petrides e Furnham (2000), com 260 estudantes universitários ingleses (média de idade igual a 22,21 anos; $D P=5,90$ ), resultou em índices muito pobres de ajuste numa Análise Fatorial Confirmatória (AFC), não confirmando a estrutura unifatorial do estudo original. Por sua vez, uma Análise Fatorial Exploratória (AFE) resultou em uma solução de quatro fatores capazes de explicar $40,4 \%$ da variância total. Eles foram interpretados como relacionados ao otimismo ou regulação do humor; avaliação das emoções, habilidades sociais e utilização de emoções. Os índices de fidedignidade das escalas não foram reportados nesse estudo. No entanto, Saklofske, Austin, e Minski (2003) replicaram o estudo de Petrides e Furnham (2000) com 354 participantes canadenses, que tinham em média 21,4 anos $(D P=4,9$ anos). A estrutura fatorial com quatro fatores foi confirmada tanto por AFE quanto por AFC. Além disso, esses fatores se juntaram em apenas um numa análise fatorial de segunda ordem. Os coeficientes alfa de Cronbach foram de 0,89, para o Fator Geral, e de $0,80,0,79,0,68$ e 0,75 , respectivamente, para os fatores otimismo ou regulação do humor, avaliação das emoções, utilização de emoções e habilidades sociais. 
O Emotional Skills and Competence Questionnaire (ESCQ) também é um inventário de autorrelato, desenvolvido originalmente na Croácia (Takšić, 1998) e posteriormente traduzido para diversos idiomas, incluindo o português europeu (Faria et al., 2006). Uma análise fatorial aplicada aos 45 itens da versão croata do instrumento $(N=2124)$ revelou uma estrutura de três fatores: percepção e compreensão de emoções (F1), expressão e nomeação de emoções (F2) e gerenciamento e regulação de emoções (F3). Correlações entre os fatores variaram de 0,35 a 0,55 , e foram interpretadas como evidências da existência de um fator geral do instrumento. Os coeficientes alfa de Cronbach desses fatores foram sempre superiores a 0,7 (Faria et al., 2006). A versão portuguesa desse instrumento foi obtida a partir de um estudo com 730 estudantes secundaristas $(52,2 \%)$ e universitários $(47,8 \%)$, cujos resultados revelaram estruturas fatoriais semelhantes entre si e à versão original croata. Os coeficientes alfa de Cronbach variaram de 0,64 no Fator $2 \mathrm{e}$ 0,90 no escore geral (Faria et al., 2006).

Medidas de autorrelato e de desempenho para avaliação da IE tendem a apresentar correlações baixas e não significativas entre si (Brackett, Mayer, \& Warner, 2004; Warwick, \& Nettelbeck, 2004) e padrões distintos de correlações com variáveis externas. Por exemplo, medidas de autorrelato tendem a se correlacionar mais com traços de personalidade e menos com habilidades cognitivas tradicionais (como o fator g) e com o desempenho educacional (como o SAT; Petrides, \& Furnham, 2003; Saklofske et al., 2003). Um padrão de inversão é encontrado em relação aos instrumentos de desempenho (por exemplo, MacCann, \& Roberts, 2008; Roberts et al., 2001;). No entanto, ambas as medidas tendem a se correlacionar com indicadores de qualidade de vida, como a satisfação com a vida ou bem-estar subjetivo (Brackett, Rivers, Shiffmann, Lerner, \& Salovey, 2006; Lopes, Salovey, \& Straus, 2003; Saklofske et al., 2003). No estudo de Saklofske et al. (2003), por exemplo, foram encontradas correlações positivas entre o fator geral de inteligência emocional (medido por autorrelato) e satisfação com a vida $(r=0,39 ; p<0,001)$ e felicidade subjetiva $(r=0,45 ; p<0,001)$, e essas correlações permaneceram significativas mesmo com o controle dos cinco grandes fatores de personalidade $(r=0,20 ; p<0,001$ e $r=0,15$; $p<0,01$, respectivamente).

Assim, considerando a falta de um instrumento de autorrelato para avaliação dessas competências emocionais no contexto cultural brasileiro e as perspectivas que seu uso em pesquisa e na prática clínica poderiam proporcionar, propõe-se a investigação das propriedades psicométricas de um Inventário de Competências Emocionais (ICE), cujo desenvolvimento se deu com base na proposta teórica de Mayer e Salovey (1997). Mais especificamente, este trabalho teve como objetivos buscar evidências de validade baseadas na estrutura interna (validade fatorial e convergente) e na relação com variáveis externas (qualidade de vida), além de investigar os índices de fidedignidade do instrumento.

\section{Método}

\section{Participantes}

A amostra foi constituída por 409 participantes, predominantemente do sexo feminino (76,5\%), com média de idade de 30,2 anos $(D P=11,5)$. A maioria era proveniente da região Nordeste $(60,4 \%)$, seguida consecutivamente por participantes das regiões Sudeste $(21,0 \%)$, Norte $(15,4 \%)$, Sul $(1,5 \%)$, Centro-Oeste $(0,2 \%)$ e estrangeiros $(1,0 \%)$. Dois participantes não informaram estado de proveniência $(0,5 \%)$. Quanto à etnia, 58,4\% dos participantes se declararam brancos; $32,5 \%$, pardos; $8,3 \%$, negros; $0,5 \%$, indígenas; e $0,2 \%$, asiáticos. A grande maioria possuía Ensino Superior completo ou incompleto $(91,9 \%)$, mas também havia participantes com Ensino Médio $(7,1 \%)$ ou Fundamental $(1,0 \%)$ completo ou incompleto. Quanto à renda, 32,2\% declararam renda inferior a 2,5 salários mínimos; $22,0 \%$, entre 2,5 e cinco salários mínimos; $22,3 \%$, entre cinco e 7,5 salários mínimos; e 23,5\%, superior a 7,5 salários mínimos.

\section{Instrumentos}

Foram aplicados três instrumentos: um questionário para coleta de informações sociodemográficas, o Inventário de Competências Emocionais, desenvolvido para este estudo, e o Inventário Breve de Qualidade de Vida da Organização Mundial de Saúde (WHOQOL). Todos os instrumentos foram formatados num formulário eletrônico do Google Drive, de modo a permitir que a coleta pudesse ser realizada pela internet.

O questionário para coleta de informações pessoais continha apenas oito questões para identificação e caracterização do participante. O Inventário de Competências Emocionais era composto por 76 frases (por exemplo, "Noto rapidamente quando um sentimento está aumentando perigosamente de intensidade"), que o participante era convidado a ler e a julgar, em uma escala Likert de cinco pontos, o quanto se aplicavam ao seu caso. Ele deveria atribuir de "1", se considerasse que "não se aplica ao meu caso", a "5", se considerasse que "se aplica perfeitamente ao meu caso".

Os itens foram elaborados pelo autor principal com base na teoria de Mayer e Salovey (1997) para representar as quatro habilidades relacionadas à inteligência emocional descritas anteriormente. Posteriormente, quatro estudantes de pós-graduação, sendo três de mestrado e um de doutorado, familiarizados com o tema em questão e que cursavam uma disciplina relacionada à construção de instrumentos de avaliação psicológica, revisaram a representatividade teórica dos itens, a existência de aspectos teóricos não contemplados por eles (lacunas) e a existência de dois ou mais itens que representassem o mesmo aspecto teórico (redundância). Não houve um registro 
formal da realização desse trabalho. As discussões aconteceram ao longo de um dia de trabalho, e as modificações ou eliminações de itens foram realizadas por consenso até a obtenção do conjunto final dos itens, que foi aplicado aos participantes.

A versão em português do Questionário de Qualidade de Vida da Organização Mundial da Saúde (WHOQOL-Bref; Fleck et al., 2000) é composta por 26 perguntas (por exemplo, "em que medida você acha que sua vida tem sentido?") para serem respondidas em uma escala Likert de cinco pontos, em que "1" significa baixa qualidade de vida, e "5" significava ótima qualidade de vida. O instrumento informa sobre qualidade de vida em quatro áreas ou domínios: físico, psicológico, relações sociais e meio-ambiente. A média das pontuações nesses quatro fatores ainda fornece um indicador da qualidade de vida de uma forma geral.

\section{Procedimentos}

Os participantes desta pesquisa foram contatados pela internet, por e-mail ou redes sociais, esclarecidos quanto aos objetivos e procedimentos da pesquisa e, os que concordaram em participar, acessaram um link disponibilizado na mensagem-convite que conduzia à página da pesquisa. Ali, eram recebidos com uma tela de agradecimento e conduzidos às páginas seguintes que continham o Termo de Compromisso Livre e Esclarecido (TCLE), no formato aprovado pelo Comitê de Ética em Pesquisa (CAAE 07085412.2.0000.5208) e os instrumentos citados anteriormente. Os dados foram armazenados automaticamente em uma planilha eletrônica e posteriormente analisados estatisticamente.

\section{Análise de Dados}

Os dados foram analisados com auxílio do Statistical Package for the Social Sciences (SPSS), versão 17, no qual foram implementadas análises fatoriais, correlacionais e de estatística descritiva para consecução dos objetivos propostos. $\mathrm{Na}$ investigação da validade fatorial, procedeu-se à extração de componentes principais com uso de rotação oblíqua mínima, uma vez que se supunha que os fatores estariam correlacionados entre si. A definição do número de fatores a serem retidos foi realizada com base numa inspeção do Gráfico de Sedimentação (Cattell, 1966), e alguns itens foram eliminados por apresentarem cargas inferiores a $0,3 \mathrm{em}$ todos os fatores. Coerentemente com a teoria, esperava-se encontrar quatro fatores, correspondentes aos propostos por Mayer e Salovey (1997): percepção de emoções, utilização da emoção para facilitação do pensamento, compreensão emocional e regulação de emoções.

Para investigação da validade convergente, foram calculados os coeficientes de correlação de Pearson entre os fatores encontrados na análise fatorial, esperando-se valores moderados e significativos entre eles. Esse padrão de resultados foi proposto e utilizado em estudos anteriores
(Mayer et al., 1999; MacCann, \& Roberts, 2008), como critério para verificação empírica da proposta de que a inteligência emocional é formada por habilidades distintas (os fatores apresentam variância única), mas relacionados ao processamento de um mesmo tipo de informação (os fatores apresentam variância compartilhada). Uma análise fatorial de segunda ordem também foi realizada, na expectativa de que todos os fatores primários formassem apenas um fator de segunda ordem, relacionado às diferenças individuais no processamento emocional global. Esses padrões de correlações e estrutura fatorial já foram encontrados em estudos anteriores com instrumentos de autorrelato (Faria et al., 2006; Takšić, 1998).

$\mathrm{Na}$ busca de evidências de validade baseada nas relações com critérios externos, esperava-se encontrar correlações moderadas, positivas e estatisticamente significativas com qualidade de vida, especialmente com o fator psicológico, conforme os resultados semelhantes obtidos nos estudos de Brackett et al. (2006); Lopes et al. (2003) e Saklofske et al. (2003). Em relação aos índices de fidedignidade, esperava-se que fossem superiores a 0,7 , conforme recomendações amplamente aceitas em estudos de construção de instrumentos (Anastasi, \& Urbina, 2000; Pasquali, 2003).

\section{Resultados}

Os resultados serão apresentados na seguinte sequência: validade fatorial e índices de fidedignidade, validade convergente e validade com critérios externos. $\mathrm{Na}$ análise fatorial, observou-se que tanto a Medida de Adequação da Amostra de Kaiser-Meyer-Olkin $(\mathrm{KMO}=0,891)$ quanto o Teste de Esfericidade de Bartlett $\left(\chi^{2}=12482,065 ; g l=2850 ; p<0,001\right)$ indicaram haver condições suficientes para a realização da análise.

Inicialmente, pelo critério de Kaiser (1960), que recomenda a retenção dos fatores com eigenvalues superiores a um, foram obtidos 20 fatores, explicando $62,4 \%$ da variância total. No entanto, com base numa inspeção do Gráfico de Sedimentação (Cattell, 1966) precedeu-se a extração de cinco fatores. No processo de análise, foram eliminados os itens 11, 13, 16 e 63, que apresentaram cargas inferiores a $0,3 \mathrm{em}$ todos os fatores. Os resultados dessa análise (cargas fatoriais e coeficientes alfa de Cronbach), encontram-se na Tabela 1.

Foi obtida uma estrutura final com cinco fatores, capazes de explicar $38,1 \%$ da variância total. Esses fatores foram interpretados como relacionados à percepção de emoções - Fator 1 (29 itens, explicando 20,3\% da variância total) -, regulação de emoções de baixa potência - Fator 2 (15 itens, 6,8\% da variância total) -, expressividade emocional - Fator 3 (oito itens, 4,3\% da variância total) -, regulação de emoções em outras pessoas Fator 4 (11 itens, 3,9\% da variância total) - e regulação de emoções de alta potência - Fator 5 (nove itens, 2,8\% da variância total). Os coeficientes alfa de Cronbach 
desses fatores variaram de 0,67 (expressividade emocional) a 0,91 (percepção de emoções), e as correlações entre eles variaram de zero (entre expressividade emocional e regulação de emoções de alta potência) a 0,576 (entre percepção de emoções e regulação de emoções em outras pessoas), com média de 0,36 .

Tabela 1

Cargas Fatoriais e Coeficientes Alfa de Cronbach

\begin{tabular}{|c|c|c|c|c|}
\hline & 1 & 2 & 4 & 5 \\
\hline $\begin{array}{l}\text { 36. Detecto a influência, positiva ou negativa, que um determinado ambiente } \\
\text { físico causa em mim. }\end{array}$ & 0,660 & & & \\
\hline $\begin{array}{l}\text { 22. Noto rapidamente quando um sentimento está aumentando perigosamente } \\
\text { de intensidade. }\end{array}$ & 0,602 & & & \\
\hline 45. Percebo quando o meu estado de humor está mudando. & 0,593 & & & \\
\hline $\begin{array}{l}\text { 15. Consigo perceber a diferença entre o que eu deveria ou gostaria de sentir e o } \\
\text { que realmente sinto numa situação. }\end{array}$ & 0,590 & & & \\
\hline 14. Sei como os meus sentimentos influenciam o meu comportamento. & 0,581 & & & \\
\hline 37. Identifico as atividades que me deixam de mau humor. & 0,571 & & & \\
\hline 60. Sei de que forma o meu estado emocional influencia aquilo que tenho que fazer. & 0,569 & & & \\
\hline $\begin{array}{l}\text { 30. Detecto a influência, positiva ou negativa, que outras pessoas exercem sobre } \\
\text { as minhas emoções. }\end{array}$ & 0,566 & & & \\
\hline $\begin{array}{l}\text { 43. Procuro relacionar os meus sentimentos com fatos anteriores que os possam } \\
\text { ter causado. }\end{array}$ & 0,560 & & & \\
\hline 75. Identifico quando uma pessoa está ou não disposta a me ajudar. & 0,528 & & & \\
\hline 44. Consigo perceber o humor das pessoas ao observá-las no dia-a-dia. & 0,523 & & & \\
\hline $\begin{array}{l}\text { 34. Percebo o impacto, positivo ou negativo, do meu comportamento sobre } \\
\text { outras pessoas. }\end{array}$ & 0,517 & & & \\
\hline 27. Noto quando estou me sentindo mal, mesmo sem saber a causa. & 0,516 & & & \\
\hline $\begin{array}{l}\text { 24. Reconheço quando os meus sentimentos comprometem uma boa tomada } \\
\text { de decisão. }\end{array}$ & 0,510 & & & \\
\hline 58. Noto quando uma pessoa está reagindo exageradamente a uma situação. & 0,492 & & $-0,335$ & \\
\hline 51. Tenho consciência de quando estou confuso em relação aos meus sentimentos. & 0,481 & & & \\
\hline $\begin{array}{l}\text { 76. Reconheço quando estou experimentando sentimentos idênticos aos de } \\
\text { outras pessoas que já passaram por experiências similares. }\end{array}$ & 0,480 & & & \\
\hline $\begin{array}{l}\text { 41. Noto quando o comportamento de uma pessoa não é compatível com aquilo } \\
\text { que ela está vivendo. }\end{array}$ & 0,479 & & & \\
\hline 32. Consigo prever como vou me sentir numa determinada situação. & 0,467 & & & \\
\hline $\begin{array}{l}\text { 68. Ao conversar com outra pessoa, detecto os sentimentos que estão por trás } \\
\text { de sua fala. }\end{array}$ & 0,461 & & $-0,339$ & \\
\hline 57. Detecto sinais emocionais sutis em outras pessoas. & 0,448 & & $-0,352$ & \\
\hline $\begin{array}{l}\text { 42. Percebo claramente o efeito que determinado filme e/ou música têm sobre } \\
\text { os meus sentimentos. }\end{array}$ & 0,444 & & & \\
\hline $\begin{array}{l}\text { 53. Procuro identificar os sentimentos que me fizeram agir de uma determinada } \\
\text { forma. }\end{array}$ & 0,443 & & & \\
\hline 64. Noto mudanças de comportamento nas pessoas com as quais convivo. & 0,422 & & $-0,337$ & \\
\hline 67. Reconheço sentimentos que já tive quando expressos por outras pessoas. & 0,421 & & & \\
\hline 2. Percebo se minhas atitudes agradam ou não às pessoas. & 0,374 & & & \\
\hline $\begin{array}{l}\text { 33. Procuro ajustar as tarefas que tenho que realizar em função do meu estado } \\
\text { emocional. }\end{array}$ & 0,349 & & & \\
\hline $\begin{array}{l}\text { 72. Sou capaz de expressar descontentamento mesmo para pessoas que são } \\
\text { importantes para mim (familiares, companheiro/a, etc.). }\end{array}$ & 0,342 & & & \\
\hline $\begin{array}{l}\text { 29. Numa conversa, procuro ver se há coerência entre o discurso e a postura } \\
\text { corporal de quem está falando. }\end{array}$ & 0,317 & & & \\
\hline 31. Sou capaz de me livrar facilmente da tristeza. & & $-0,748$ & & \\
\hline
\end{tabular}


Tabela 1 (continuação)

Cargas Fatoriais e Coeficientes Alfa de Cronbach

\begin{tabular}{|c|c|c|c|c|c|}
\hline & 1 & 2 & 3 & 4 & 5 \\
\hline 55. Consigo vencer o meu desânimo. & & $-0,691$ & & & \\
\hline 26. Frustrações deixam-me desanimado/a por bastante tempo. & & 0,675 & & & \\
\hline 23. Sou capaz de me motivar novamente quando algo na minha vida não sai bem. & & $-0,661$ & & & \\
\hline 4. Sei o que devo fazer para me manter num bom estado de espírito. & & $-0,531$ & & & \\
\hline $\begin{array}{l}\text { 52. Quando estou com um sentimento negativo, sei o que devo fazer para me } \\
\text { sentir melhor. }\end{array}$ & & $-0,507$ & & & \\
\hline 66. Tenho dificuldades para confrontar outra pessoa quando o desejo fazer. & & 0,497 & & & \\
\hline 18. Consigo vencer os meus medos. & & $-0,458$ & & & \\
\hline 20. Conheço meios para melhorar meu estado emocional. & & $-0,441$ & & & \\
\hline 46. Me chateio quando alguém não me dá a atenção que eu esperava ter. & & 0,391 & & & \\
\hline $\begin{array}{l}\text { 10. Percebo que os meus sentimentos se manifestam desproporcionalmente a } \\
\text { uma dada situação. }\end{array}$ & & 0,388 & & & \\
\hline 59. Sei dizer "não" a outras pessoas. & & $-0,377$ & & & \\
\hline $\begin{array}{l}\text { 7. Sei como gerar em mim mesmo o sentimento apropriado para aquilo que } \\
\text { tenho que fazer. }\end{array}$ & & $-0,375$ & & & \\
\hline 50. Receio tornar-me vulnerável ao expressar os meus sentimentos. & & 0,338 & 0,333 & & \\
\hline 39. Procuro priorizar as atividades que melhoram o meu estado de humor. & 0,313 & $-0,317$ & & & \\
\hline 69. Sou emocionalmente expressivo. & & & $-0,659$ & & \\
\hline 9. Tenho facilidade de expressar o que sinto. & & & $-0,633$ & & \\
\hline 28. Sou capaz de expressar os meus sentimentos de apreço por alguém. & & & $-0,561$ & & \\
\hline 56. Tenho vergonha de expressar os meus sentimentos. & & & 0,521 & & \\
\hline 71. As minhas decisões são tomadas friamente. & & & 0,518 & & \\
\hline 3. Considero meus sentimentos ao tomar alguma decisão. & & & $-0,432$ & & \\
\hline $\begin{array}{l}\text { 70. Consigo expressar o oposto do que estou sentindo, caso isso seja } \\
\text { socialmente mais apropriado numa situação. }\end{array}$ & & & 0,395 & & \\
\hline 47. Se eu quiser, consigo controlar completamente a minha expressão emocional. & & & 0,379 & & \\
\hline 54. Sei como acalmar uma pessoa nervosa. & & & & $-0,766$ & \\
\hline 65. Sei como motivar as pessoas para darem o melhor de si. & & & & $-0,757$ & \\
\hline 49. Consigo ajudar outras pessoas a se sentirem melhor. & & & & $-0,719$ & \\
\hline 62. Sei como encorajar uma pessoa a enfrentar o seu medo. & & & & $-0,712$ & \\
\hline 25. Sei como motivar uma pessoa desanimada, sem constrangê-la. & & & & $-0,675$ & \\
\hline 17. Sei como acalmar uma pessoa eufórica, sem desanimá-la. & & & & $-0,667$ & \\
\hline 6. Tenho jeito para lidar com pessoas problemáticas. & & & & $-0,518$ & 0,348 \\
\hline $\begin{array}{l}\text { 48. Consigo prever como pessoas que conheço bem vão reagir numa } \\
\text { determinada situação. }\end{array}$ & & & & $-0,393$ & \\
\hline 74. Consigo descobrir o que motiva as pessoas próximas a mim. & & & & $-0,373$ & \\
\hline $\begin{array}{l}\text { 38. Ao conversar com uma pessoa que acabei de conhecer, sei como me } \\
\text { relacionar com ela. }\end{array}$ & & & & $-0,361$ & \\
\hline 1. Consigo contagiar as pessoas com a emoção que estou sentindo. & & & & $-0,349$ & \\
\hline $\begin{array}{l}\text { 61. Evito deixar que o meu mau humor influencie o meu relacionamento com } \\
\text { as pessoas. }\end{array}$ & & & & & 0,518 \\
\hline 73. "Conto até dez" para não agir descontroladamente. & & & & & 0,510 \\
\hline 40. Consigo controlar a minha irritação. & & & & & 0,491 \\
\hline 5. Por mais que tente, não consigo controlar a expressão do que estou sentindo. & & & & & $-0,477$ \\
\hline 8. Procuro compreender a causa emocional dos comportamentos das pessoas. & & & & & 0,468 \\
\hline 21. Consigo expressar o meu descontentamento de forma apropriada. & & & & & 0,442 \\
\hline
\end{tabular}


Tabela 1 (continuação)

Cargas Fatoriais e Coeficientes Alfa de Cronbach

\begin{tabular}{|c|c|c|c|c|c|}
\hline & 1 & 2 & 3 & 4 & 5 \\
\hline $\begin{array}{l}\text { 19. Quando estou tomado(a) pela emoção, procuro afastar-me da situação até } \\
\text { que possa encará-la de modo mais sereno. }\end{array}$ & & & & & 0,439 \\
\hline 12. Coloco-me no lugar das pessoas para compreender os seus sentimentos. & & & & & 0,415 \\
\hline 35. Consigo controlar-me para não agir sob influência da euforia, quando necessário. & & & & & 0,369 \\
\hline Coeficientes alfa de Cronbach & 0,911 & 0,831 & 0,670 & 0,863 & 0,766 \\
\hline
\end{tabular}

Dada a possibilidade de extração de um fator de segunda ordem, uma nova análise fatorial foi implementada, com extração dos fatores por componentes principais e rotação oblíqua. Foram obtidos dois fatores capazes de explicar $69,3 \%$ da variância total. O primeiro fator de segunda ordem (Fator Geral de Competências Emocionais), cujas cargas variaram de 0,74 (Fator 2, regulação de emoções de baixa potência) a 0,93 (Fator 4, regulação de emoções em outras pessoas), só não incluiu o Fator 3 (expressividade emocional), que ficou isolado no segundo fator de segunda ordem (carga igual a 0,96). $\mathrm{O}$ coeficiente alfa de Cronbach do primeiro fator foi de 0,92 . As estatísticas descritivas de todos os fatores são apresentadas na Tabela 2 .

Tabela 2

Estatísticas Descritivas do Inventário de Competências Emocionais

\begin{tabular}{lcccc}
\hline \multicolumn{1}{c}{ Fatores } & M & DP & Simetria & Curtose \\
\hline F1 - Percepção de Emoções & 4,06 & 0,49 & $-0,353$ & $-0,245$ \\
F2 - Regulação de Emoções de Baixa Potência & 3,21 & 0,63 & 0,002 & $-0,455$ \\
F3 - Expressividade Emocional & 3,58 & 0,64 & $-0,400$ & $-0,070$ \\
F4 - Regulação de Emoções em Outras Pessoas & 3,64 & 0,67 & $-0,364$ & $-0,119$ \\
F5 - Regulação de Emoções de Alta Potência & 3,40 & 0,67 & $-0,273$ & $-0,263$ \\
Fator Geral de Competências Emocionais & 3,58 & 0,48 & $-0,025$ & $-0,322$ \\
\hline
\end{tabular}

Nota-se que as médias ficaram pouco acima do ponto central da escala de cinco pontos, com exceção do primeiro fator, cuja média avançou para a categoria 4. Os valores de simetria e curtose não se distanciaram muito de zero, mas confirmam a média acima do ponto central pela obtenção de simetria negativa.

Finalmente, para investigar a relação das competências emocionais com um critério externo (qualidade de vida), foram calculados os coeficientes de correlação de Pearson entre os fatores de ambos os instrumentos. Os resultados estão na Tabela 3 .

Nota-se que os fatores relacionados à regulação de emoções apresentaram um padrão de correlações mais consistentes, tanto em quantidade quanto em intensidade, com os vários aspectos da qualidade de vida. Destacase a regulação de emoções de baixa potência, que se correlacionou de forma mais elevada e com todos os fatores de qualidade de vida, atingindo a magnitude de 0,610 para o aspecto psicológico.

Tabela 3

Coeficientes de Correlação de Pearson

\begin{tabular}{lccccc}
\hline & Físico & Psicológico & Relações Sociais & Meio-ambiente & QL Geral \\
\hline F1 & 0,05 & $0,18^{*}$ & 0,13 & $0,16^{*}$ & 0,10 \\
F2 & $0,33^{* *}$ & $0,61^{* *}$ & $0,43^{* *}$ & $0,32^{* *}$ & $0,24^{* *}$ \\
F3 & 0,00 & 0,06 & $0,19^{*}$ & 0,15 & 0,01 \\
F4 & 0,10 & $0,23^{* *}$ & $0,32^{* *}$ & $0,24^{* *}$ & 0,01 \\
F5 & $0,18^{*}$ & $0,28^{* *}$ & $0,34^{* *}$ & 0,14 & 0,03 \\
CE Geral & $0,23^{* *}$ & $0,43^{* *}$ & $0,41^{* *}$ & $0,28^{* *}$ & 0,12 \\
\hline
\end{tabular}

Nota. F1 - Percepção de Emoções, F2 - Regulação de Emoções de Baixa Potência, F3 - Expressividade Emocional, F4 - Regulação de Emoções em Outras Pessoas e F5 - Regulação de Emoções de Alta Potência. *Valores estatisticamente significativos ao nível de 0,05, bicaudal. ${ }^{* *}$ Valores estatisticamente significativos ao nível de 0,01 , bicaudal. 
Há, portanto, dados relacionados à estrutura fatorial do instrumento, fidedignidade dos fatores encontrados e relação com critérios externos. Esses dados parecem apontar tanto para a obtenção de um instrumento com boas propriedades psicométricas quanto para possibilidade de refiná-lo para obtenção de uma segunda versão mais eficiente.

\section{Discussão}

Este trabalho teve o objetivo de buscar evidências de validade baseadas na estrutura interna (fatorial e convergente) e na relação com variáveis externas (qualidade de vida) para um Inventário de Competências Emocionais (ICE). Os cinco fatores obtidos na análise fatorial não são exatamente os esperados, mas são compatíveis com a proposta teórica de Mayer e Salovey (1997). O primeiro fator reuniu afirmações que foram construídas para avaliação da percepção de emoções em si e em outras pessoas (por exemplo, "Detecto a influência, positiva ou negati$\mathrm{va}$, que um determinado ambiente físico causa em mim", ou "Identifico quando uma pessoa está ou não disposta a me ajudar"), da utilização das emoções para facilitação do pensamento (por exemplo, "Sei de que forma o meu estado emocional influencia aquilo que tenho que fazer") e do conhecimento emocional (por exemplo, "Procuro relacionar os meus sentimentos com fatos anteriores que os possam ter causado"). Ainda que esses itens tenham sido construídos para avaliação de habilidades distintas, é possível identificar neles, especialmente nos que obtiveram cargas mais elevadas, conteúdos relacionados à percepção de emoções. Por exemplo, os verbos que predominam nas frases são: detecto, noto, percebo, identifico, reconheço, tenho consciência, entre outros.

Além disso, ele é compatível com o fator de avaliação das emoções encontrado no Emotional Intelligence Inventory (Petrides, \& Furnham, 2000; Saklofske et al, 2003) e com uma parte dos itens dos fatores de percepção e compreensão de emoções (F1) e expressão e nomeação de emoções (F2) do Emotional Skills and Competence Questionnaire (ESCQ; Faria et al., 2006). Assim, esse fator foi identificado como compatível às habilidades associadas à percepção de emoções por Mayer e Salovey (1997) e assim denominado.

$O$ fato de esse fator ter reunido itens que inicialmente estavam previstos para outros fatores acabou resultando em um número de itens muito elevado. Isso sugere a necessidade de estudos posteriores tanto para tentar representar melhor os aspectos que não resultaram em fatores no instrumento (nomeadamente a utilização da emoção para facilitação do pensamento e a compreensão de emoções), como para reduzir o número de itens do primeiro fator.

Todas as afirmações do segundo fator estão relacionadas à regulação de emoções (por exemplo, "Sou capaz de me livrar facilmente da tristeza").
No entanto, referem-se ao controle de emoções desagradáveis (valência negativa), que causam sensações de baixa energia e desmotivação e impelem o indivíduo a se retirar ou fugir do ambiente (baixa potência) (Fontaine, Scherer, Roesch, \& Ellsworth, 2007). Esse fator, portanto, identifica uma nuance da habilidade de regular emoções da proposta de Mayer e Salovey (1997), referindo-se especificamente à regulação de emoções de baixa potência (em oposição ao Fator 5, que se refere à regulação de emoções de alta potência). Esse fator também é compatível com otimismo e regulação do humor, no Emotional Intelligence Inventory (Petrides, \& Furnham, 2000; Saklofske et al., 2003), e com gerenciamento e regulação de emoções, no Emotional Skills and Competence Questionnaire (ESCQ; Faria et al., 2006).

O terceiro fator reuniu oito itens relacionados predominantemente à expressividade emocional (por exemplo, "sou emocionalmente expressivo"), e é parcialmente compatível com o fator denominado expressão e nomeação de emoções encontrado no Emotional Skills and Competence Questionnaire (ESCQ; Faria et al., 2006). A expressividade emocional foi incluída no primeiro nível hierárquico da inteligência emocional, proposto por Mayer e Salovey (1997), juntamente com a percepção de emoções. No entanto, nunca foi contemplada em instrumentos de avaliação da IE por desempenho, construídos por esses autores (MEIS, MSCEIT). Nesse sentido, a obtenção desse fator mostra que a avaliação por autorrelato pode acessar, com facilidade, habilidades cuja avaliação por desempenho seria muito mais complicada do ponto de vista da captação e da avaliação da resposta.

O quarto fator ficou claramente relacionado à regulação de emoções em outras pessoas (por exemplo, "Sei como acalmar uma pessoa nervosa"). Esse fator se aproxima em instrumentos de autorrelato baseados em outras concepções de inteligência emocional (cuja descrição extrapola os objetivos deste trabalho), como os aspectos interpessoais do Bar-On Emotional Quotient Inventory (EQi; BarOn, 1997), ou o quociente emocional interpessoal encontrado no estudo de Palmer Manocha, Gignac, e Stouch, (2003) com o mesmo EQi, mas é mais restrito porque se refere somente ao aspecto de modificação do estado emocional de outra pessoa, e não ao relacionamento interpessoal como um todo. Por isso, esse fator foi denominado de regulação de emoções em outras pessoas.

Uma análise do conteúdo dos itens do quinto fator também o associou à regulação de emoções, mas, nesse caso os itens se referem a emoções que poderiam ser identificadas no trabalho de Fontaine et al. (2007) como de valência negativa e arousal e potência elevadas. Essas características emocionais fazem o indivíduo sentir-se dono de si, com poder e energia para transformar motivação em ação, na direção do contato com o objeto de interesse. Portanto, esse fator se diferencia do segundo por se referir ao controle de emoções de alta potência, como a raiva e euforia, por exemplo, que motivam 
comportamentos por impulso ("Consigo controlar a minha irritação", "Consigo controlar-me para não agir sob influência da euforia, quando necessário”). Por isso, esse fator se relaciona a outras escalas de forma semelhante ao segundo fator descrito anteriormente, e foi denominado de regulação de emoções de alta potência.

$\mathrm{Na}$ sequência, foram investigadas as consistências internas dos fatores obtidos pelos coeficientes alfa de Cronbach, cuja variação reflete, em parte, o número de itens desses fatores. Mas, de forma geral, índices podem ser considerados muito bons, permitindo explicar as diferenças individuais pelos construtos avaliados, e não por outras variáveis aleatórias (Anastasi, \& Urbina, 2000; Pasquali, 2003). Assim, em resumo, foram identificados os seguintes fatores: percepção de emoções, F1 $(\alpha=0,911)$; regulação de emoções de baixa potência, F2 $(\alpha=0,831)$; expressividade emocional, F3 $(\alpha=0,670)$; regulação de emoções em outras pessoas, F4 $(\alpha=0,863)$; e regulação de emoções de alta potência, F5 $(\alpha=0,766)$.

Conforme o esperado, esses fatores apresentaram um padrão de correlações positivas entre si de magnitudes moderadas a altas, com exceção do Fator 3 (expressividade emocional), que apresentou correlações baixas com os demais fatores. Os resultados da análise fatorial de segunda ordem confirmaram o padrão correlacional e permitiram a obtenção de uma pontuação geral mais relacionada à percepção e regulação de emoções (o Fator Geral de Competências Emocionais). Esses dados podem ser interpretados como uma evidência de validade convergente para o instrumento, já que fatores relacionados com o mesmo construto convergem. A exceção ficou por conta da expressividade emocional, que, contraditoriamente à proposição teórica (Mayer, \& Salovey, 1997), não convergiu para as demais habilidades relacionas ao processamento emocional. Duas hipóteses podem ser levantadas para essa ocorrência: 1. os itens não representam bem a expressividade emocional; 2. a expressividade emocional é uma habilidade que não deve ser incluída na inteligência emocional. É mais provável que a primeira hipótese seja verdadeira, pois, embora os itens com carga mais elevada estejam relacionados com a expressividade emocional, há outros itens que guardam pouca relação com essa habilidade e podem estar prejudicando a relação com as outras habilidades (por exemplo, "Tenho vergonha de expressar os meus sentimentos", "As minhas decisões são tomadas friamente", "Considero meus sentimentos ao tomar alguma decisão"). No entanto, os dados obtidos neste estudo não apoiam essa inclusão, em decorrência da obtenção de um fator de segunda ordem somente com o fator primário de expressividade emocional.

Na observação das estatísticas descritivas dessas escalas, notou-se a tendência de as médias ficarem um pouco acima do ponto central da escala de cinco pontos. É possível que esse fato seja devido à desejabilidade social do conteúdo descrito nos itens de competência emocional.
No entanto, considerando os valores de simetria e curtose, que sugerem que as distribuições dos escores se aproximam de uma curva normal, pode-se concluir que a escala de cinco pontos foi suficiente e adequada para que os participantes expressassem suas características.

Finalmente, na busca de evidências de validade com base nas relações com variáveis externas, foi identificada uma relação mais estreita da qualidade de vida com as variantes da regulação de emoções, sendo que a regulação de emoções de baixa potência foi a que se correlacionou mais robustamente com todos os aspectos da qualidade de vida, especialmente com o psicológico. Embora este estudo seja do tipo correlacional, que não permite o estabelecimento de relações causais, esse resultado ao menos aponta a possibilidade de que a regulação de emoções tenha um papel importante na obtenção de uma qualidade de vida, sugerindo a necessidade de realizar outras pesquisas que permitam investigar se essa relação é causal. Isso teria uma importante implicação para programas de intervenção em diversos contextos de atuação do psicólogo, pois o desenvolvimento de estratégias de regulação de emoções em geral e de emoções de baixa potência em particular, poderia resultar na melhoria da qualidade de vida de uma forma geral, incluindo aspectos mais distantes do funcionamento psicológico, como o físico e o meio-ambiente. Além disso, esse conjunto de dados pode ser considerado como uma evidência de validade baseada nas relações com variáveis externas (nesse caso, a qualidade de vida) para o Inventário de Competências Emocionais.

Esse conjunto de resultados permite atestar a validade fatorial e de critério (para qualidade de vida) do Inventário de Competências Emocionais. Embora nem todos os fatores tenham sido representados (faltaram os fatores de utilização da emoção para facilitação do pensamento e compreensão de emoções), o que sugere a necessidade de revisão e refinamento do instrumento, pode-se dizer que os fatores encontrados são compatíveis com a teoria da inteligência emocional proposta por Mayer e Salovey (1997). Este estudo também acrescenta dados que sugerem que: (a) por autorrelato, é possível obter um fator relacionado à expressividade emocional (fator que nunca foi representado em instrumentos por desempenho), embora sua pertinência à inteligência emocional tenha que ser verificada em estudos futuros; (b) a regulação de emoções apresentou nuances importantes, indicando que provavelmente haja processos mentais distintos para o controle de emoções de baixa e de alta potência em si mesmo e em outras pessoas.

Uma limitação deste estudo é a predominância de participantes com bom nível de escolaridade (com Ensino Superior completo ou incompleto), o que facilita a realização da tarefa de leitura, compreensão e avaliação das afirmações, favorecendo também a obtenção de fatores válidos e precisos. Além disso, não foi feito 
nenhum controle sobre o funcionamento psicológico dos participantes, supondo-se que a amostra seja composta, em sua maioria, por pessoas que não apresentam transtornos mentais importantes. Esses fatos sugerem a necessidade de estudos posteriores com populações de nível de escolaridade mais baixo ou com transtornos mentais importantes, para verificar o funcionamento do instrumento e sua utilidade nessas condições mais adversas. Apesar disso, este estudo apresenta um instrumento válido e fidedigno para avaliação de competências emocionais, que pode ser recomendado para utilização em pesquisas.

\section{Referências}

Anastasi, A., \& Urbina, S. (2000). Testagem psicológica. Porto Alegre: Artes Médicas.

Bar-On, R. (1997). Bar-On Emotional Quotient Inventory (EQi): Technical manual. Toronto, Canada: Multi-Health Systems.

Brackett, M. A., Mayer, J. D., \& Warner, R. M. (2004). Emotional intelligence and its relation to everyday behaviour. Personality and Individual Differences, 36, 1387-1402.

Brackett, M. A., Rivers, S. E., Shiffman, S., Lerner, N., \& Salovey, P. (2006). Relating emotional abilities to social functioning: A comparison of self-report and performance measures of emotional intelligence. Journal of Personality and Social Psychology, 91(4),780-795.

Bueno, J. M. H. (2013). Construção e validação de um instrumento para avaliação da regulação emocional. Estudos Interdisciplinares em Psicologia, 4(2), 186-200.

Bueno, J. M. H., \& Primi, R. (2003). Inteligência emocional: um estudo de validade sobre capacidade de perceber emoções. Psicologia: Reflexão e Crítica, 16(2), 279-291.

Cattell, R. B. (1966). The scree test for the number of factors. Multivariate Behavioral Research, 1(2), 245-276.

Chico, E. (1999). Evaluación psicometrica de una escala de inteligencia emocional. Boletin de Psicologia Spain, 62, 65-78.

Cobêro, C. (2004). Inteligência emocional: validade do MSCEIT no contexto organizacional. (Dissertação de mestrado não publicada), Universidade São Francisco, Itatiba, SP, Brasil

Dantas, M. A., \& Noronha, A. P. P. (2005). Inteligência emocional: parâmetros psicométricos de um instrumento de medida. Estudo Pesquisa em Psicologia, 5(1), 59-72.

Faria, L., Santos, N. L., Takšić, V., Räty, H., Molander, B., Holmström, S., \& Toyota, H. (2006). Cross-cultural validation of the Emotional Skills and Competence Questionnaire (ESCQ). Psicologia, 20(2), 95-127.

Fleck, M. P. A., Louzada, S., Xavier, M., Chachamovich, E., Vieira, G., Santos, L., \& Pinzon, V. (2000). Aplicação da versão em português do instrumento abreviado de avaliação da qualidade de vida "WHOQOL-Brief". Saúde Pública, 34(2), 178-183.

Fontaine, J. R. J.; Scherer, K. R., Roesch, E. B., \& Ellsworth, P. C. (2007)). The world of emotions is not two-dimensional. Psychological science, 18(12), 1050-1057.

Hedlund, J., \& Sternberg, R. J. (2002). Inteligências em excesso? Integrando as inteligências social, emocional e prática (R. C. Costa, Trad.). Em R. Bar-On, \& J. D. A. Parker (Orgs.), Manual de inteligência emocional: teoria, desenvolvimento, avaliação e aplicação em casa, na escola e no local de trabalho (pp. 111-131). Porto Alegre: Artmed. (Original publicado em 2000).

Jesus Junior, A. G. (2004). Estudo de validade e precisão do Mayer-Salovey-Caruso Emotional Intelligence Test. (Dissertação de mestrado não publicada), Universidade São Francisco, Itatiba, SP, Brasil.

Kaiser, H. F. (1960). The application of electronic-computers to factor-analysis. Educational and Psychological Measurement, 20(1), $141-151$.

Lopes, P. N., Salovey, P., \& Straus, R. (2003). Emotional intelligence, personality, and the perceived quality of social relationships. Personality and Individual Differences, 35, 641-658.

MacCann, C., \& Roberts, R. D. (2008). New paradigms for assessing emotional intelligence: Theory and data. Emotion, 8(4), $540-551$.

Mayer, J. D., \& Salovey, P. (1997). What is emotional intelligence? Em: P. Salovey, \& D. J. Sluyter (Eds.). Emotional development and emotional intelligence: Implications for educators (pp. 3-31). New York, Basic Books.

Mayer, J. D., Salovey, P., \& Caruso, D. R. (1997). Emotional IQ test (CD ROM), Needham, MA: Virtual Knowledge.

Mayer, J. D., Salovey, P., \& Caruso, D. R. (1999). Emotional intelligence meets standards for an intelligence. Intelligence, 27(4), $267-298$.

Mayer, J. D., Salovey, P., \& Caruso, D. R. (2000). Emotional Intelligence as zeitgeist, as personality, and as mental ability. Em R. Bar-On, \& D. A. J. Parker (Orgs.), The handbook of emotional intelligence: theory development, assessment, and applications at home, school, and in the workplace (pp. 92-117). San Francisco: Jossey-Bass Inc.

Mayer, J. D., Salovey, P., \& Caruso, D. R. (2002). Mayer-Salovey-Caruso Emotional Intelligence Test (MSCEIT) User's manual. Toronto, Canada: MHS Publishers.

Mayer, J. D., Salovey, P., Caruso, D. R., \& Sitarenios, G. (2003). Measuring emotional intelligence with the MSCEIT V2.0. Emotion, 3(1), 97-105.

Mayer, J. D.; Roberts, R. D., \& Barsade, S. G. (2008). Human abilities: Emotional intelligence. Annual Review of Psychology, 59 , $507-36$.

Noronha, A. P. P., Primi, R., Freitas, F. A., \& Dantas, M. A. (2007). Análise dos itens do Mayer-Salovey-Caruso Emotional Intelligence Test: escalas da área estratégica. Psicologia em Estudo, 12(2), 415-422.

Palmer, B. R., Gignac, G., Manocha, R., \& Stough, C. (2005). A psychometric evaluation of the Mayer-Salovey-Caruso Emotional Intelligence Test Version 2.0. Intelligence, 33(3), 285-305.

Palmer, B. R., Manocha, R., Gignac, G., \& Stough, C. (2003). Examining the factor structure of the BarOn Emotional Quotient Inventory with an Australian general population sample. Personality and Individual Differences, 35(5), 1191-1210.

Pasquali, L. (2003). Psicometria: teoria dos testes na Psicologia e na educação. Petrópolis: Vozes.

Petrides, K. V., \& Furnham, A. (2000). On the dimensional structure of emotional intelligence. Personality and Individual Differences, 29(2), 313-320.

Petrides, K. V., \& Furnham, A. (2003). Trait emotional intelligence: Behavioural validation in two studies of emotion recognition and reactivity to mood induction. European Journal of Personality, 17, 39-57. 
Primi, R., Bueno, J. M. H., \& Muniz, M. (2006). Inteligência emocional: validade convergente e discriminante do MSCEIT com a BPR-5 e o 16PF. Psicologia Ciência e Profissão, 26(1), 26-45.

Roberts, R. D; Zeidner, M., \& Matthews, G. (2001). Does emotional intelligence meet traditional standards for intelligence? Some new data and conclusions. Emotion, 1(3), 196-231.

Saklofske, D. H.; Austin, E. J., \& Minski, P. S. (2003). Factor structure and validity of a trait emotional intelligence measure. Personality and Individual Differences, 34(4), 707-721.

Salovey, P., \& Grewal D. (2005). The science of emotional intelligence. Current Directions in Psychological Science, 14(6), $281-285$.

Salovey, P., \& Mayer, J. D. (1990). Emotional intelligence. Imagination, Cognition and Personality, 9, 185-211.

Schutte, N. S., Malouff, J. M, Hall, L. E., Haggerty, D. J., Cooper, J. T., Golden, C. J., \& Dornheim, L. (1998). Development and validation of a measure of emotional intelligence. Personality and Individual Differences, 25(2), 167-177.

Takšić, V. (1998). Validacija konstrukta emocionalne inteligencije. [Validation of the Emotional Intelligence Construct]. (Tese de doutorado não publicada), University of Zagreb, Croácia.

Warwick, J., \& Nettelbeck, T. (2004). Emotional intelligence is. ...? Personality and Individual Differences, 37, 1091-1100.

Woyciekoski, C., \& Hutz, C. S. (2009). Inteligência emocional: teoria, pesquisa, medida, aplicações e controvérsias. Psicologia: Reflexão e Crítica, 22(1), 1-11.

\section{Sobre os autores}

José Maurício Haas Bueno é Doutor em Psicologia pelo Programa de Pós-Graduação Stricto Sensu em Psicologia da Universidade São Francisco. Atualmente é Professor do Programa de Pós-Graduação em Psicologia Cognitiva da Universidade Federal de Pernambuco.

Fernanda Maria de Lira Correia é aluna de graduação em Psicologia na Universidade Federal de Pernambuco, bolsista de iniciação científica pelo CNPq.

Mussa Abacar possui Mestrado pela Universidade do Porto. Atualmente é Docência da Universidade Pedagogica Moçambique e doutorando pelo Programa de Pós-Graduação em Psicologia Cognitiva da Universidade Federal de Pernambuco..

Yves de Albuquerque Gomes é Doutor pelo Programa de Pós-Graduação em Psicologia Cognitiva da Universidade Federal de Pernambuco.

Francisco Santos Pereira Júnior é Mestre pelo Programa de Pós-Graduação em Psicologia Cognitiva da Universidade Federal de Pernambuco. 